\title{
Could Dystonia Be Initial Presentation of Corpus Callosum Infarction in Young Age Patients? A Case Report Study
}

\author{
Mohamed Hamdy Ibrahim1, Alyaa Fadhil2, Sameh Saied Ali3 , Salma Fathy Abdel Kader3, \\ Mohamed Khalid ${ }^{4}$, Kiran Kumar ${ }^{4}$, Shivram Kumar5, Janhavi Sirsat ${ }^{5}$ \\ ${ }^{1}$ Department of Neurology, GMCH \& RC, Ajman, United Arab Emirates \\ ${ }^{2}$ Department of Clinical Pharmacology, GMCH \& RC, Ajman, United Arab Emirates \\ ${ }^{3}$ Department of Radiology, GMCH \& RC, Ajman, United Arab Emirates \\ ${ }^{4}$ Department of Internal Medicine, GMCH \& RC, Ajman, United Arab Emirates \\ ${ }^{5}$ Gulf Medical University (GMU), Ajman, United Arab Emirates \\ Email: mohamedhamdy neuro2007@yahoo.com
}

Received 31 March 2015; accepted 6 June 2015; published 10 June 2015

Copyright (C) 2015 by authors and Scientific Research Publishing Inc.

This work is licensed under the Creative Commons Attribution International License (CC BY).

http://creativecommons.org/licenses/by/4.0/

(c) () Open Access

\section{Abstract}

Focal dystonia in young aged patients is considered to be the uncommon clinical presentation, unless a secondary cause is to be considered. Infarcts of the corpus callosum are rare and have not been well documented previously. As for a variety of signs and symptoms due to corpus callosum lesion, focal dystonia can be easily overlooked. The case is approved by ethical committee and explained to the patient with patient approval.

Keywords

Focal Dystonia, Corpus Callosum Infarction, Involuntary Movement

\section{Introduction}

Infarcts of the corpus callosum have not been well documented in the radiologic literature. They are not common [1]. This is most likely due to a rich blood supply from three main arterial systems, especially the anterior cerebral, anterior communicating, and posterior cerebral arteries [2]. Corpus callosum infarcts may present with slowly evolving and/or non-localizing neurologic signs and symptoms that suggest the diagnosis of tumor rather than infarct. In addition, they may exhibit radiologic features more often associated with neoplasm, such as masslike enhancement or extension across the midline, which prompt biopsy. The clinical manifestation of the 
acute corpus callosum infarction is lack of specificity and complex because it often merges with other location infarction [1] [3]. Thus, it is easily missed diagnosis in the early stage [4]. With the widespread application of nuclear magnetic resonance (NMR), its diagnostic rate is much higher [2] [5] [6].

\section{Case Presentation}

A male patient 19 years old, dental student presented to the neurology clinic by sudden onset of dystonic posturing in the trunk with flexion of the trunk on speaking and frequent spontaneous myoclonic jerks at rest, continue during sleep too.

On examination: General examination showed no abnormalities, Neurological examination showed, Dystonia and frequent myoclonic jerks, staccato speech, dysphagia to fluids. With no other cranial nerves abnormalities.

Patient was hospitalized, MRI brain and MRA showed focus of subtle high T2 and FLAIR signals with evidence of diffusion restriction at the splenium of the corpus callosum (Figure 1), ECHO cardiogram was done to detected possible cardiac source of infarction and it showed him to have normal ECHO findings. Complete blood count (CBC) was normal. Protein C is low as 53\% (reference is 67 - 195) and Protein S is low as $23 \%$ (Reference is 55\% - 123\%).

Patient was advised to go for transesophegeal ECHO, and Antithrombin III level in blood.

During hospitalization, he was given the followings; Clonazepam $1 \mathrm{mg}$ every 12 hours, lamotrogine 100 mg/12hours, levodopa 12, 5 mg half tablet every 12 hours, clopidogrel $75 \mathrm{mg} / 24$ hour.

Patient showed dramatic improvement as regard the involuntary movements.

\section{Discussion}

Infarcts of the corpus callosum are not common attributed to its rich blood supply from three main arterial systems: the anterior communicating artery, the pericallosal artery, and the posterior pericallosal artery [7]. A detailed description of the vascular supply to the corpus callosum was published by Ture et al. [8].

The corpus callosum (CC) is the largest fiber bundle that connects cortical and subcortical regions of the brain [9]. It also interconnects both cerebral hemispheres, promoting functional integration of sensory and motor functions [10]. It is anatomically divided into rostrum, genu, body and splenium. The corpus callosum plays an integral role in replaying sensory, motor and cognitive information from homologous regions in the two cerebral hemispheres. Lesions of the corpus callosum can present a diagnostic dilemma, both for the radiologist and the clinician. Clinically, they are associated with neuropsychiatric symptoms, mainly interhemispheric disconnection syndromes. Patients may experience gait disorders, apraxia, agraphia, tactile anomia, alien hand syndrome and so on [11] [12].

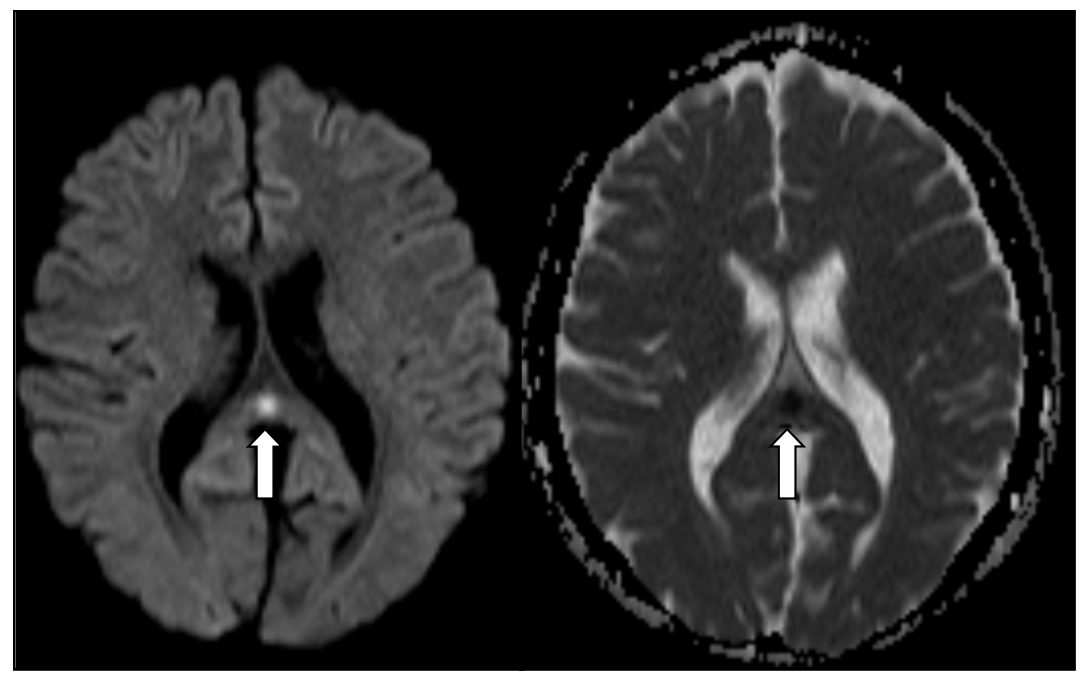

Figure 1. MRI diffusion-weighted image and the corresponding ADC showing focus of true diffusion restriction areas seen in splenium of corpus callosum impressive of acute lacunar infarct. 


\section{Conclusions}

In conclusion, the corpus callosum infarction is lack of typical symptoms and signs because it often occurs with other cerebral ischemic lesion. It is reminded of callosal infarction when the patient has consciousness or cognitive change, apraxia, such as alien hand syndrome with mild paralysis. The patients are at higher risk of stroke and the main risk factors are carotid intima thickening or plaque formation, hypertension, hyperlipidemia and the cerebral artery stenosis. The hemodynamic disorder after the cerebral large vascular lesions may be the main pathogenesis. Transcranial Doppler TCD and cervical Doppler ultrasound examination can find early hemodynamic disorder to guide the prevention and treatment.

Protein $\mathrm{C}$ and $\mathrm{S}$ deficiencies could be important causes of stroke in young age.

\section{References}

[1] Murthy, S.B., Chmayssani, M., Shah, S., Goldsmith, C.E. and Kass, J.S. (2013) Clinical and Radiologic Spectrum of Corpus Callosum Infarctions: Clues to the Etiology. Journal of Clinical Neuroscience, 20, 175-177.

http://dx.doi.org/10.1016/j.jocn.2012.05.013

[2] Yuan, J.L., Wang, S.K., Guo, X.J. and Hu, W.L. (2011) Acute Infarct of the Corpus Callosum Presenting as Alien Hand Syndrome: Evidence of Diffusion Weighted Imaging and Magnetic Resonance Angiography. BMC Neurology, 9, 142. http://dx.doi.org/10.1186/1471-2377-11-142

[3] Ishizaki, M., Ueyama, H., Nishida, Y., Imamura, S., Hirano, T. and Uchino, M. (2012) Crossed Aphasia Following an Infarction in the Right Corpus Callosum. Clinical Neurology and Neurosurgery, 114, 161-165.

http://dx.doi.org/10.1016/j.clineuro.2011.09.009

[4] Goenka, A.H., Mukund, A., Ahuja, J. and Kumar, A. (2010) Reversible Lesion in the Splenium of the Corpus Callosum in a Child with Influenza-Associated Encephalitis, Encephalopathy (IAEE). Journal of Clinical Neuroscience, 17, 607, 678.

[5] Kang, K. and Choi, N.C. (2012) Ipsilateral Hemiparesis and Spontaneous Horizontal Nystagmus Caused by Middle Cerebral Artery Territory Infarct in a Patient with Agenesis of the Corpus Callosum. Neurological Sciences, 33, 11651168. http://dx.doi.org/10.1007/s10072-011-0871-2

[6] Zhang, L.L., Tang, Q., Wang, Z. and Zhang, X.S. (2012) Alveolar Soft Part Sarcoma of the Uterine Corpus with Pelvic Lymph Node Metastasis: Case Report and Literature Review. International Journal of Clinical and Experimental Pathology, 5, 715-719.

[7] Saito, Y., Matsumura, K. and Shimizu, T. (2006) Anter-Ograde Amnesia Associated with Infarction of the Anterior Fornix and Genu of the Corpus Callosum. Journal of Stroke \& Cerebrovascular Diseases, 15, 176-177. http://dx.doi.org/10.1016/j.jstrokecerebrovasdis.2006.04.004

[8] Ture, U., Yasargil, M.G. and Krisht, A.F. (1996) The Arteries of the Corpus Callosum: A Microsurgical Anatomic Study. Neurosurgery, 39, 1075-1085. http://dx.doi.org/10.1097/00006123-199612000-00001

[9] Aloumanis, K.P. and Papapetrou, P.D. (2007) Corpus Callosum Aplasia in a Young Patient with a Parathyroid Adenoma. Journal of Clinical Neuroscience, 11, 1124-1126. http://dx.doi.org/10.1016/j.jocn.2006.01.020

[10] Hofer, S. and Frahm, J. (2006) Topography of the Human Corpus Callosum Revisited: Comprehensive Fiber Tractography Using Diffusion Tensor Magnetic Resonance Imaging. NeuroImage, 32, 989-994. http://dx.doi.org/10.1016/j.neuroimage.2006.05.044

[11] Shin, H.W., Song, S.K. and Sohn, Y.H. (2013) Is Progressive Upper-Body Apraxia a Corticobasal Syndrome? Journal of Clinical Neuroscience, 20, 319-322. http://dx.doi.org/10.1016/j.jocn.2012.02.029

[12] Nakamura, T. and Fujishima, I. (2013) Usefulness of Ice Massage in Triggering the Swallow Reflex. Journal of Stroke \& Cerebrovascular Diseases, 22, 378-382. http://dx.doi.org/10.1016/j.jstrokecerebrovasdis.2011.09.016 